\title{
A Study to Assess the Prevalence of Text Neck Syndrome and Quality of Sleep among Smartphone Users in Selected Colleges of District Ludhiana, Punjab
}

\author{
Amninder Kaur ${ }^{1}$, Simran Makker ${ }^{2}$ \\ ${ }^{1}$ Assistant Professor in Department of Community Health Nursing, DMCH College of Nursing, Ludhiana, \\ Punjab, India \\ ${ }^{2}$ Bachelor of Science in Nursing student, DMCH College of Nursing, Ludhiana, Punjab, India
}

Corresponding Author: Amninder Kaur

\begin{abstract}
Background of Study: Text neck syndrome is an important health issues especially in developing Countries. Text Neck refers to repetitive stress injury resulted from excessive watching, texting or overusing hand held devices in a forward head posture for a long period of time. The prevalence of smartphone users worldwide will be around 2.5 billion by 2019. In 2019, the smartphone users in India are estimated to hit 340 million. However, the health effects of mobile phone usage were various on mental and physical health system, but the problem out of which cervical, neck pain and insomnia was most commonly reported.

Objectives: To assess the prevalence of Text Neck Syndrome and Quality of Sleep among smartphone users in selected colleges of District Ludhiana, Punjab.

Material and Methods: A descriptive research design was used. Purposive sampling technique was used to select 400 smartphone users. Data was gathered by using standardized tool for text neck syndrome i.e. Neck Disability Index and Pittsburgh Sleep Quality Index to assess quality of sleep. Data was analyzed by using descriptive and inferential statistics.

Results: The results of study showed that nearly half of the smartphone users 151(37.8\%) had mild neck disability and $(53.75 \%)$ subjects had good sleep quality whereas $(46.25 \%)$ poor sleep quality. There was a statistical significant association of the text neck syndrome with sociodemographic variable habitat of smart phone users and association of sleep quality with habitat, socioeconomic status and academic course.

Conclusion: The study findings revealed that nearly $37.8 \%$ had mild neck disability and $(53.75 \%)$ subjects had good sleep quality among smartphone users and was significantly associated with habitat.
\end{abstract}

Keywords: Text neck syndrome, Quality of Sleep, smartphone users, repetitive stress injury

\section{INTRODUCTION}

The prevalence of smartphone users worldwide surpasses 3.2 billion by 2019 . China (851.15) and India (345.92) ranked as first and second as per the number of smartphone users.

Text Neck Syndrome refers to repetitive stress injury resulted from excessive watching, texting or overusing hand held devices in a forward head posture for a long period of time.

As per the reports of research studies about health effects of mobile phone usage were various on mental and physical health system, but the problem out of which neck pain(73.8\%), ringing in ears $(72.93 \%)$, 
headache $(64.63 \%)$ and sleeping disorders $(58.52 \%)$ was most commonly reported

Poor sleep quality is affecting $10 \%$ to $50 \%$ of smart phone users. Poor sleep quality consequences are many and have a profound impact on the smartphone users' physical and psychological health.

\section{MATERIAL \& METHODS}

A descriptive research design was used to assess prevalence of text neck syndrome among 400 smart phone users selected by purposive sampling technique. The sample size was calculated. The inclusion criteria for smart phone users was falling in the age group of 18-25 years, using smartphone more than one hour a day, able to understand the English language. The exclusion criteria for smart phone users who had any congenital, traumatic \& pathological cervical diagnosed health problem. Data was collected from smart phone users by self report method after getting ethical permission from Dayanand Medical College Hospital as well as Principals of respective colleges from Satish Chander Dhawan (SCD) Government College and Arya College, Civil lines Ludhiana, Punjab with standardized tool developed by Dr. Howard (1980) for measuring self-rated disability due to neck pain and how it had affected the everyday activities of smart phone users and Pittsburgh Sleep Quality index (PSQI, 1988) to assess Quality of sleep. Data was analyzed by using descriptive and inferential statistics as per objectives of study.

\section{RESULTS}

As per socio demographic characteristics the majority of $89.2 \%$ smartphone users were in the age group of 18-21 years, $68.2 \%$ were male, more than half $54.8 \%$ were commerce students, maximum $73.2 \%$ smart phone users live in the urban area,70\% smart phone users belong to Hindu religion $70 \%$.

$61.5 \%$ of smart phone users were from nuclear family, $98.2 \%$ smart phone users lives at home. As per socio-economic status more than half $62.2 \%$ of the smart phone users were from middle class family.

Table1: Frequency \& percentage Distribution of smartphone user as per their smart phone usage profile, $N=400$

\begin{tabular}{|c|c|c|}
\hline $\begin{array}{l}\text { Sr } \\
\text { No. }\end{array}$ & Smartphone Usage Profile & $\mathbf{f}(\%)$ \\
\hline 1. & \multicolumn{2}{|l|}{ Smartphone usage hours } \\
\hline & $\begin{array}{l}1-2 \\
3-4 \\
5-6 \\
>6 \\
\text { Mean } \pm \mathrm{SD}=2.00 \pm 096 \\
\end{array}$ & $\begin{array}{l}145(36.2) \\
151(37.8) \\
63(15.8) \\
41(10.2)\end{array}$ \\
\hline 2. & \multicolumn{2}{|c|}{ Common application used in smartphone } \\
\hline & $\begin{array}{l}\text { Searching } \\
\text { Games } \\
\text { Academic } \\
\text { Scientific } \\
\text { Others }\end{array}$ & $\begin{array}{l}211(52.8) \\
88(22.0) \\
24(6.0) \\
22(5.5) \\
55(13.8)\end{array}$ \\
\hline 3. & \multicolumn{2}{|c|}{ Common mode of communication } \\
\hline & $\begin{array}{l}\text { Texting } \\
\text { Video chatting } \\
\text { Phone calling } \\
\text { Others }\end{array}$ & $\begin{array}{l}211(52.8) \\
88(22.0) \\
24(6.0) \\
77(19.2)\end{array}$ \\
\hline 4. & \multicolumn{2}{|c|}{ Posture use while using smartphone } \\
\hline & $\begin{array}{l}\text { Flexed neck } \\
\text { Standing } \\
\text { Sitting } \\
\text { Lying }\end{array}$ & $\begin{array}{l}73(18.2) \\
16(4.0) \\
218(54.5) \\
93(23.2)\end{array}$ \\
\hline 5. & \multicolumn{2}{|c|}{ Hours spend on smartphone other than calls (In Hours) } \\
\hline & $\begin{array}{l}<1 \\
1-2 \\
3-4 \\
>4 \\
\text { Mean } \pm \mathrm{SD}=1.88 \pm 0.92\end{array}$ & $\begin{array}{l}172(43.0) \\
129(32.2) \\
73(18.2) \\
26(6.5)\end{array}$ \\
\hline 6. & \multicolumn{2}{|c|}{$\begin{array}{l}\text { Experienced neck \& shoulder pain while using } \\
\text { smartphone }\end{array}$} \\
\hline & $\begin{array}{l}\text { No } \\
\text { I don't know } \\
\text { Just happen sometime } \\
\text { It is continue }\end{array}$ & $\begin{array}{l}188(47.0) \\
41(10.2) \\
156(39.0) \\
15(3.8)\end{array}$ \\
\hline 7. & \multicolumn{2}{|c|}{ Taking break while using smartphone } \\
\hline & $\begin{array}{l}\text { No break } \\
\text { Break every } 20 \text { minutes } \\
\text { Break every } 30 \text { minutes } \\
\text { Break every } 1 \text { hour }\end{array}$ & $\begin{array}{l}72(18.0) \\
104(26.0) \\
92(23.0) \\
132(33.0)\end{array}$ \\
\hline 8. & \multicolumn{2}{|l|}{ Hours sleep during the day } \\
\hline & $\begin{array}{l}<1 \\
1-2 \\
3-4 \\
>4\end{array}$ & $\begin{array}{l}118(29.5) \\
129(32.25) \\
64(16.0) \\
89(22.25) \\
\end{array}$ \\
\hline 9. & \multicolumn{2}{|c|}{ Using mobile phone at bed time in night } \\
\hline & $\begin{array}{l}\text { Yes } \\
\text { No }\end{array}$ & $\begin{array}{l}273(68.2) \\
127(31.7)\end{array}$ \\
\hline & \multicolumn{2}{|c|}{ If, yes how many hours using smartphone $(n=273)^{*}$} \\
\hline & $\begin{array}{l}1 \text { hour } \\
2-3 \text { hours } \\
3-4 \text { hours } \\
>4 \text { hours }\end{array}$ & $\begin{array}{l}197(49.2) \\
53(13.2) \\
13(3.2) \\
10(2.5)\end{array}$ \\
\hline 10. & \multicolumn{2}{|c|}{ Time taken by you to fall asleep } \\
\hline & $\begin{array}{l}<15 \text { minutes } \\
16-30 \text { minutes } \\
31-60 \text { minutes } \\
>60 \text { minutes }\end{array}$ & $\begin{array}{l}201(50.2) \\
129(32.2) \\
48(12.0) \\
22(5.5)\end{array}$ \\
\hline 11. & \multicolumn{2}{|c|}{ Checking smartphone during sleep } \\
\hline & $\begin{array}{l}\text { Yes } \\
\text { No }\end{array}$ & $\begin{array}{l}127(31.8) \\
273(68.2)\end{array}$ \\
\hline
\end{tabular}

Table 1 interprets smartphone usage profile $(37.8 \%)$ smartphone users uses for 3- 
Amninder Kaur et.al. A study to assess the prevalence of text neck syndrome and quality of sleep among smartphone users in selected colleges of district Ludhiana, Punjab.

4 hours for searching, (52.8\%) Half of the students used texting as a mode of communication, $(52.8 \%)$ in sitting posture $(54.5 \%),(43 \%)$ smartphone users spend less than 1 hour on smartphone other than calls. (68.2\%) maximum number of smartphone users were using smartphone at bedtime in night for 1 hour (49.2\%) and more than half of the smartphone users (68.2\%) do not check their smart phone during sleep.

Table 2 Prevalence of text neck syndrome among smartphone users as per neck disability index, $\mathrm{N}=400$

\begin{tabular}{|l|l|l|}
\hline Sr. no. & Neck Disability & f (\%) \\
\hline 1 & Present & $217(54.25)$ \\
\hline 2 & Absent & $183(45.8)$ \\
\hline
\end{tabular}

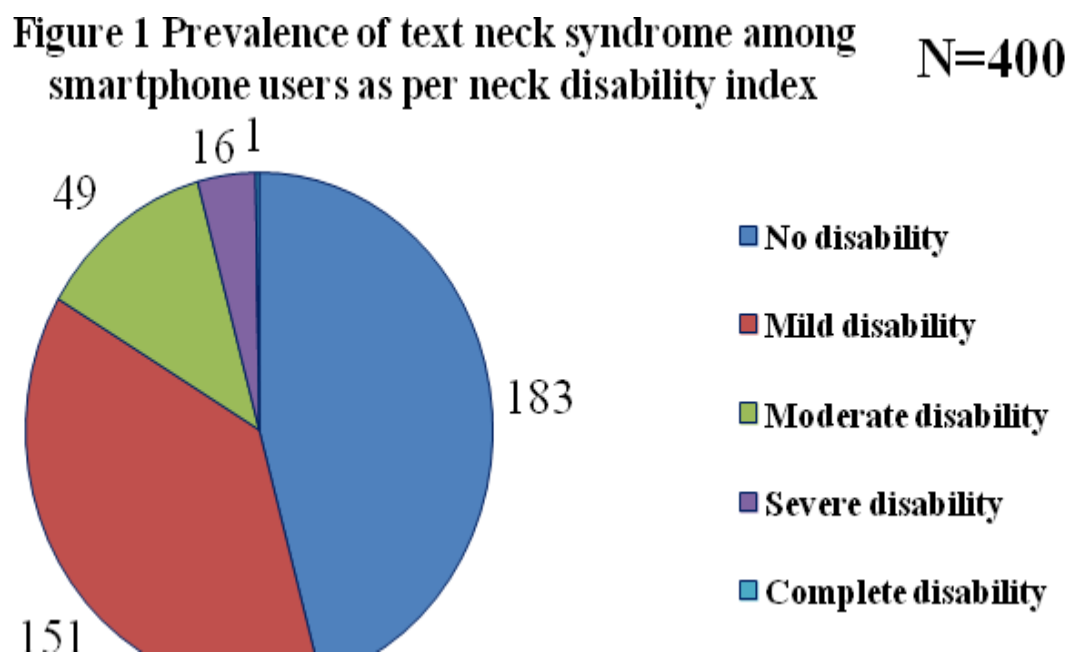

* Mean $\pm S D=7.33 \pm 7.34$

Figure 1 illustrates that 183(45.8\%) smart phone users had no neck disability, followed by $151(37.8 \%)$ mild disability, $49(12.2 \%)$ moderate, 16(4\%) severe disability and only $1(0.2 \%)$ smart phone user reported with complete neck disability.

\section{Quality of sleep among smartphone users}

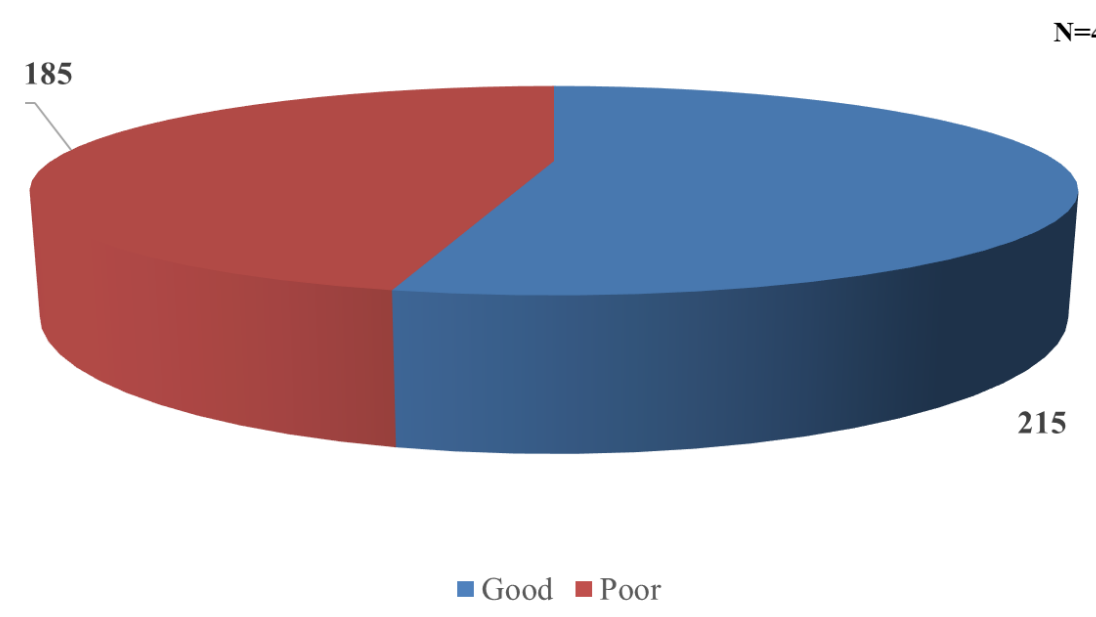

Fig 2: Quality of sleep among smartphone users as per Pittsburgh sleep quality index.

Table 2 Clarify that there was significant association of text neck syndrome with habitat of smart phone users. 
Amninder Kaur et.al. A study to assess the prevalence of text neck syndrome and quality of sleep among smartphone users in selected colleges of district Ludhiana, Punjab.

Table 2 Association of text neck syndrome with selected sociodemographic characteristics among smart phone users, $\mathrm{N}=400$

\begin{tabular}{|c|c|c|c|c|c|}
\hline Sr. No & Socio-demographic characteristics & $\mathbf{n}$ & Mean \pm SD & t/F value & p value \\
\hline \multirow[t]{2}{*}{1.} & Age(in years) & & & & \\
\hline & $\begin{array}{l}18-21 \\
22-25\end{array}$ & $\begin{array}{l}357 \\
43\end{array}$ & $\begin{array}{l}7.43 \pm 7.50 \\
43 \pm 6.37\end{array}$ & 0.788 & $0.375 \mathrm{NS}$ \\
\hline \multirow[t]{2}{*}{2.} & Gender & & & & \\
\hline & $\begin{array}{l}\text { Male } \\
\text { Female }\end{array}$ & $\begin{array}{l}273 \\
127 \\
\end{array}$ & $\begin{array}{l}7.01 \pm 7.27 \\
7.97 \pm 7.50 \\
\end{array}$ & 1.651 & $0.200 \mathrm{NS}$ \\
\hline \multirow[t]{2}{*}{4.} & Habitat & & & & \\
\hline & $\begin{array}{l}\text { Rural } \\
\text { Urban }\end{array}$ & $\begin{array}{l}107 \\
292\end{array}$ & $\begin{array}{l}9.38 \pm 9.39 \\
6.57 \pm 6.30\end{array}$ & 29.545 & $0.000^{*}$ \\
\hline \multirow[t]{2}{*}{3.} & Academic course & & & & \\
\hline & $\begin{array}{l}\text { Medical } \\
\text { Non-medical } \\
\text { Commerce } \\
\text { Arts }\end{array}$ & $\begin{array}{l}37 \\
22 \\
219 \\
122\end{array}$ & $\begin{array}{l}9.86 \pm 9.67 \\
9.00 \pm 7.73 \\
6.94 \pm 7.12 \\
6.90 \pm 6.74\end{array}$ & 2.207 & $0.087 \mathrm{NS}$ \\
\hline \multirow[t]{2}{*}{5.} & Type of family & & & & \\
\hline & $\begin{array}{l}\text { Joint } \\
\text { Nuclear }\end{array}$ & $\begin{array}{l}154 \\
246\end{array}$ & $\begin{array}{l}7.78 \pm 7.83 \\
7.02 \pm 7.02\end{array}$ & 1.807 & $0.180 \mathrm{NS}$ \\
\hline \multirow[t]{2}{*}{6.} & Student residential status & & & & \\
\hline & $\begin{array}{l}\text { Home } \\
\text { PG }\end{array}$ & $\begin{array}{l}391 \\
7\end{array}$ & $\begin{array}{l}7.35 \pm 7.38 \\
6.29 \pm 6.23\end{array}$ & 0.565 & $0.453 \mathrm{NS}$ \\
\hline \multirow[t]{2}{*}{7.} & Religion & & & & \\
\hline & $\begin{array}{l}\text { Sikh } \\
\text { Muslim } \\
\text { Hindu } \\
\text { Christian } \\
\text { Others } \\
\end{array}$ & $\begin{array}{l}108 \\
9 \\
280 \\
1 \\
2\end{array}$ & $\begin{array}{l}8.19 \pm 8.39 \\
11.56 \pm 7.76 \\
6.86 \pm 6.88 \\
5.00 \pm . \\
5.50 \pm 3.53\end{array}$ & 1.457 & $0.215 \mathrm{NS}$ \\
\hline \multirow[t]{2}{*}{8.} & Socio-economic status(Kuppuswamy's scale 2019) & & & & \\
\hline & $\begin{array}{l}\text { Upper-class I } \\
\text { Upper middle class II } \\
\text { Middle class III } \\
\text { Lower middle class IV } \\
\text { Lower class V }\end{array}$ & $\begin{array}{l}46 \\
55 \\
249 \\
40 \\
10\end{array}$ & $\begin{array}{l}6.93 \pm 7.34 \\
7.47 \pm 6.80 \\
7.35 \pm 7.45 \\
8.20 \pm 8.10 \\
3.80 \pm 3.85\end{array}$ & 0.753 & $0.557 \mathrm{NS}$ \\
\hline
\end{tabular}

Table3: Association of quality of sleep with selected socio-demographic characteristics among smartphone users. $\mathrm{N}=400$

\begin{tabular}{|c|c|c|c|c|c|}
\hline Sr. No & Socio-demographic characteristics & $\mathbf{n}$ & Mean \pm SD & t/F value & p value \\
\hline \multirow[t]{2}{*}{1.} & Age(in years) & & & & \\
\hline & $\begin{array}{l}18-21 \\
22-25\end{array}$ & $\begin{array}{l}357 \\
43\end{array}$ & $\begin{array}{l}4.92 \pm 3.030 \\
4.70 \pm 3.181\end{array}$ & 0.197 & $0.657 \mathrm{NS}$ \\
\hline \multirow[t]{2}{*}{2.} & Gender & & & & \\
\hline & $\begin{array}{l}\text { Male } \\
\text { Female }\end{array}$ & $\begin{array}{l}273 \\
127\end{array}$ & $\begin{array}{l}4.70 \pm 3.077 \\
5.30 \pm 2.942\end{array}$ & 0.180 & $0.672 \mathrm{NS}$ \\
\hline \multirow[t]{2}{*}{3.} & Academic course & & & & \\
\hline & $\begin{array}{l}\text { Medical } \\
\text { Non- medical } \\
\text { Commerce } \\
\text { Arts }\end{array}$ & $\begin{array}{l}37 \\
22 \\
219 \\
122\end{array}$ & $\begin{array}{l}6.08 \pm 4.304 \\
5.64 \pm 2.821 \\
4.66 \pm 2.734 \\
4.82 \pm 3,091\end{array}$ & 2.815 & $0.039 *$ \\
\hline \multirow[t]{2}{*}{4.} & Habitat & & & & \\
\hline & $\begin{array}{l}\text { Rural } \\
\text { Urban }\end{array}$ & $\begin{array}{l}107 \\
293 \\
\end{array}$ & $\begin{array}{l}5.82 \pm 3.916 \\
4.54 \pm 2.577 \\
\end{array}$ & 35.592 & $0.000 *$ \\
\hline \multirow[t]{2}{*}{5.} & Type of the family & & & & \\
\hline & $\begin{array}{l}\text { Joint } \\
\text { Nuclear }\end{array}$ & $\begin{array}{l}154 \\
246\end{array}$ & $\begin{array}{l}5.08 \pm 3.148 \\
4.77 \pm 2.977\end{array}$ & 0.990 & $0.320 \mathrm{NS}$ \\
\hline \multirow[t]{2}{*}{6.} & Student residential status & & & & \\
\hline & $\begin{array}{l}\text { Home } \\
\text { PG }\end{array}$ & $\begin{array}{l}391 \\
9\end{array}$ & $\begin{array}{l}4.90 \pm 3.031 \\
4.57 \pm 4.077\end{array}$ & 1.507 & $0.220 \mathrm{NS}$ \\
\hline \multirow[t]{2}{*}{7.} & Religion & & & & \\
\hline & $\begin{array}{l}\text { Sikh } \\
\text { Muslim } \\
\text { Hindu } \\
\text { Christian }\end{array}$ & $\begin{array}{l}108 \\
280 \\
9 \\
3\end{array}$ & $\begin{array}{l}5.68 \pm 3.312 \\
5.44 \pm 2.698 \\
4.60 \pm 2.903 \\
2.50 \pm 0.707\end{array}$ & 3.108 & $0.015^{*}$ \\
\hline \multirow[t]{2}{*}{8.} & Socio-economic status (As per Kuppuswamy scale 2019) & & & & \\
\hline & $\begin{array}{l}\text { Upper-class I } \\
\text { Upper-middle class II } \\
\text { Middle class III } \\
\text { Lower middle class IV } \\
\text { Lower class V }\end{array}$ & $\begin{array}{l}6 \\
55 \\
249 \\
40 \\
10\end{array}$ & $\begin{array}{l}4.33 \pm 3.334 \\
5.07 \pm 2.356 \\
4.89 \pm 2.950 \\
4.88 \pm 3.864 \\
2.70 \pm 2.406\end{array}$ & 2.837 & $0.024 *$ \\
\hline
\end{tabular}


Table 3 interprets that there was significant association of quality of sleep with socio-demographic characteristics like habitat, religion, academic course and economic status of smartphone users

\section{DISCUSSION}

The finding of present study revealed that out of 400 smartphone users most of the smartphone users 151(37.8\%) had mild disability followed by moderate disability 49(12.2\%).Similar results that (48\%) physiotherapy students had mild neck disability were reported by Shah PP, Sheth MS (2018) conducted a study on prevalence of text neck syndrome and SMS thumb among physiotherapy students in Gujarat.

Samuel John Asir, Pokhral Nikita, Akter Rahemun, Sohel Ahmed (2019) reported in their research study that $(46.9 \%)$ college students had pain in their neck, $(42.5 \%)$ subjects were having mild to severe disability in their neck due to smartphone.

Omar Samarah, Mohamad Yasin, Tareq Kanaan (2019) conducted a study on association between mobile phone use and neck pain among university students the results of study interpreted that neck pain severity showed that age $(\mathrm{p}=0.04)$ and duration of use $(\mathrm{p}=0.001)$ were significantly associated with the severity of neck pain, while only the duration of use was significantly associated with pain duration $(p=0.036)$. Subjects were divided into two groups according to the pain score, $75.8 \%$ had pain severity equal or less than $4 / 10$ and $24.2 \%$ had pain severity more than $4 / 10$. Of those with pain severity $>4,5.8 \%$ of students sought medical help at the emergency department and $12.4 \%$ visited clinics, compared to only $0.3 \%$ seeking medical advice at an emergency department and $4.2 \%$ visiting clinics in the group with pain severity of $\leq 4(p<0.001)$.

In Present study results it was revealed that out of 400 students $(53.75 \%)$ had good sleep quality and $(46.25 \%)$ had poor sleep quality. Whereas Similar results were reported by a study on the effect of smartphone usage at bedtime on sleep quality among Saudi Non- Medical staff at King Saudi University by Fahdah A Alshobaili, Nada A Yousefi. in 2019 that $(41.7 \%)$ of participants have poor quality of sleep due to usage of smartphone.

In current study it was found that there was significant association of sleep quality with habitat, socioeconomic status and academic course. On the other hand contrary results were reported by study Ghoreishi et al. (2018) on university medical students. Results revealed that there is no significant association of quality of sleep with socioeconomic status, habitat.

\section{CONCLUSION}

The study findings revealed that nearly $37.8 \%$ had mild neck disability among smartphone users. Near to half $(46.25 \%)$ had poor quality of sleep The results of study revealed that the mobile phone users experience subjective symptoms, the intensity of which depends upon intensity of use of mobile phones. We recommend to minimize its adverse effect by use of hand free devices, maintaining proper posture while texting and avoiding use of mobile phone during sleep.

\section{ACKNOWLEDGMENT}

We are thankful to Dayanand Medical College Hospital College of Nursing for believing in us to conduct this research study with their due ethical consideration as well as Principals of respective colleges from Satish Chander Dhawan (SCD) Government College and Arya College, Civil lines Ludhiana, Punjab as well as their students who were part of this study for giving us consent to give their valuable time and research related required information.

Conflict of Interest: None

Source of Funding: None

Ethical Approval: Approved 


\section{REFERENCES}

1. Shah PP, Sheth MS. Correlation of smartphone use addiction with text neck syndrome and SMS thumb in physiotherapy students. Int $\mathrm{J}$ Community med public health. 2018; 5(6): 2512- 2516.

2. Shrivastava A, Sexena Y, effect of mobile usage on serum melatonin levels among medical students. Indian $\mathbf{J}$ physiol pharmacol 2014; 58(4): 395-399

3. Statista Research Department. India mobile phone users [updated 2015 Aug. 31; cited 2019 July 30]. Available from: https// www. Statista.com / statistics / 274658.

4. Kim MS. Influence of neck pain on cervical movement in the sagittal plane during smartphone use. J Phys Ther Sci. 2015 Jan; 27(1): 15-17. doi: 10.1589/jpts.27.15

5. Robles P, South china morning post. New York spine surgery and rehabilitation medicine. Int $\mathrm{J}$ of interdisciplinary research; 2019

6. Samami PP, et al. Awareness of text neck syndrome in young adult population. Int $\mathbf{J}$ community Med public Health. 2018; 5(8).
7. Athavale NA, P et al SP. Awareness of text neck syndrome in young - adult population. (Accessed 2018 June 12) Available from http:// dx.doi.org / 10. 18203/2394-6040).

8. Bader K AlZarea, Santosh R Patil. Mobile phone head and neck pain syndrome proposal of a new entity. OHDM. 2015; 14(5); 313-317.

9. Abdulrahman NA, Prevalence of neck pain and relationship between use of electronic device and neck pain 2017.( accessed 2018 January 30); 70(11); 1992-1999.

10. Sung - JA KOO. Effect of duration of smartphone use on muscle fatigue and pain caused by forward head posture 2016; J. Phys. The sci. 28(6); 1669-1672.

How to cite this article: Kaur A, Makker S. A study to assess the prevalence of text neck syndrome and quality of sleep among smartphone users in selected colleges of district Ludhiana, Punjab. Int J Health Sci Res. 2021; 11(9): 49-54. DOI: https://doi.org/10.52403/ ijhsr.20210907 